\title{
Evaluation of Analgesic Activity of Some Novel Quinazolinone Analogues
}

\author{
Ritesh Patel $^{1 *}$, Rohit Saraswat ${ }^{2}$ and Sujit Pillai ${ }^{3}$ \\ ${ }^{1}$ Indore Institute of Pharmacy, Indore, MP, India \\ ${ }^{2}$ Department of Pharmacy, OPJS University, Churu, RJ, India \\ ${ }^{3} G R Y$ Institute of Pharmacy, Khargone, MP, India \\ *Corresponding Author: Ritesh Patel, Indore Institute of Pharmacy, Indore, MP, \\ India.
}

Received: April 11, 2020

Published: May 04, 2020

(c) All rights are reserved by PRitesh Patel.,

et al.

\begin{abstract}
The synthesis, characterization and spectroscopic studies of new quinazolinone substituted analogues $Q_{1}-Q_{16}$ with analgesic activity were described. A series of novel quinazolinone derivatives were synthesized. In this view, 5-chloro anthranilic acid undergoes acetylation in the presence of acetic anhydride and anhydrous sodium acetate to give 5-chloro- $\mathrm{N}$-acetyl anthranilic acid as intermediate-I which upon cyclization in the presence of phosphorous pentaoxide, glacial acetic acid and para amino benzoic acid to yield 4-[6-chloro-2-methyl-4-oxoquinazolin-3(4H)-yl] benzoic acid as intermediate-II. This resulted intermediate-II undergo mannich base reaction to produce novel quinazolinone derivatives on reaction of formaldehyde with different aromatic amines. All the synthetic derivatives were fully characterized by spectral analytical data (elemental analysis, FTIR, 1H NMR and Mass) and the purity of the compounds was determined by TLC. Analgesic activities were tested via both hot plate and acetic acid induced writhing methods. The study concluded that the compound $Q_{5}, Q_{8}$ and $Q_{9}$ were found to exhibit significant analgesic activity when compared to Ibuprofen as standard drug while other derivatives exhibit moderate to good analgesic activity.

Keywords: 5-Chloro Anthranilic Acid; Quinazolinone; Analgesic Activity; Ibuprofen
\end{abstract}

\section{Introduction}

It is evident from literature that, Quinazolinone is a heterocyclic compound play vital role towards synthetic medicinal chemistry. The synthetic derivatives of quinazolinone are utilized as therapeutic agent for combating against different pathological conditions. 5-chloro anthranilic acid mainly employed for the synthesis of quinazolinone compounds as starting materials [1]. Quinazolinone and its derivatives possess a major class of biologically active compounds which exhibited large spectrum of therapeutic activities including; anti-malarial [2], analgesic [3], antioxidant [4], anticancer [5], antiviral [6], antifeedant [7], sedative-hypnotic [8], anticonvulsant [9], antimicrobial [10], antialgal [11], hypotensive [12] and anti-inflammatory [13]. Recently quinazolinone derivatives seek great attention of researchers in organic and medicinal chemistry due to their prompt biological activities. Encouraged by the therapeutic diversity of quinazolinone containing moiety and the comparative ease of convertibility of anthranilic acid to quinazolinone, we took up the synthesis of certain novel quinazolinone from 5-chloro anthranilic acid and evaluated their analgesic activity [14].

\section{Materials and Methods}

All the chemicals used in the synthesis of the intermediates and final derivatives were of A.R grade and procured from the Merck and LOBA chemicals. All the synthesized quinazolinone derivatives were characterized by melting point determination using Veergo digital melting point apparatus in open capillary tubes and were uncorrected.
IR Spectra were recorded using Perkin Elmer FTIR spectrophotometer using $\mathrm{KBr}$ pellets techniques and ${ }^{1} \mathrm{HNMR}$ spectra of the synthesized compounds in deuterated DMSO were recorded on BRUKER AVANCE II 400MHz NMR Spectrometer instrument using TMS as the internal standard. Mass Spectra were recorded using LC-MSD-Tranp-SL2010A SHIMADZU using Dimethylsulphoxide (DMSO) as solvent. TLC was performed using silica gel $\mathrm{GF}_{254}$ coated plates of $0.25 \mathrm{~mm}$ thickness. Ethyl acetate, petroleum ether, chloroform (0.6:0.8:8.6) were used as solvent system and iodine vapors as visualizing agent.

Scheme of synthesis

(Figure and Table 1)

The experimental work comprises in three steps:

1. Step-I: Synthesis of 5-chloro-N-acetyl anthranilic acid from 5 -chloro anthranilic acid.

2. Step-II: Synthesis of 4-[6-chloro-2-methyl-4-oxo quinazolin-3(4H)-yl] benzoic acid.

3. Step-III: Synthesis of various derivatives of quinazolinone by mannich reaction.

Step-I: General procedure for the synthesis of 5-chloro-N-acetyl anthranilic acid from 5 -chloro anthranilic acid (Intermediate-I)

5-Chloro anthranilic acid ( 0.02 moles) was mixed with an equimolar quantities of anhydrous sodium acetate ( 0.03 moles) and 


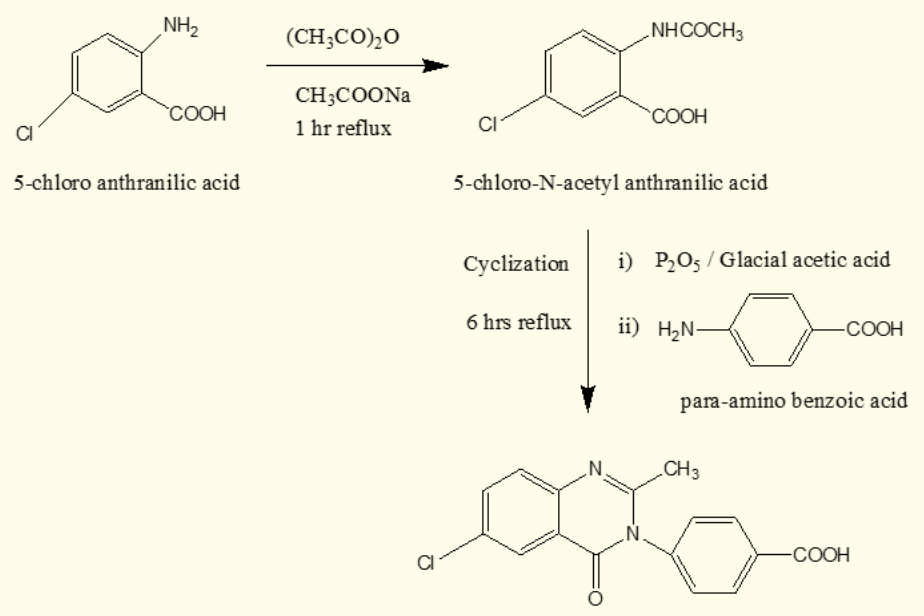

4-[6-chloro-2-methyl-4-oxoquinazolin-3(4H)-yl]benzoic acid
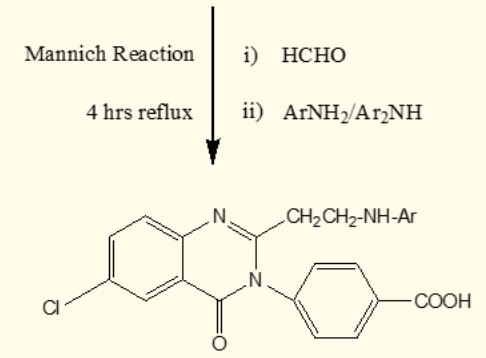

4-[6-chloro-2-arylaminoethyl-4-oxoquinazolin-3(4H)-yl]benzoic acid

$\mathrm{Ar}=$ DIFFERENT AROMATIC AMINES

Figure

\begin{tabular}{|c|c|c|c|}
\hline S. No. & Compounds Code & Substituted Aromatic Amine (Ar) & Structure of Aromatic Amine (Ar) \\
\hline 1 & $\mathrm{Q}_{1}$ & Aniline & \\
\hline 2 & $\mathrm{Q}_{2}$ & o-nitro aniline & \\
\hline 3 & $\mathrm{Q}_{3}$ & m-nitro aniline & \\
\hline 4 & $\mathrm{Q}_{4}$ & p-nitro aniline & \\
\hline 5 & $Q_{5}$ & o-bromo aniline & \\
\hline 6 & $\mathrm{Q}_{6}$ & m-bromo aniline & \\
\hline 7 & $\mathrm{Q}_{7}$ & p-bromo aniline & \\
\hline 8 & $\mathrm{Q}_{8}$ & o-chloro aniline & \\
\hline
\end{tabular}




\begin{tabular}{|c|c|c|c|}
\hline 9 & $\mathrm{Q}_{9}$ & m-chloro aniline & \\
\hline 10 & $\mathrm{Q}_{10}$ & p-chloro aniline & \\
\hline 11 & $\mathrm{Q}_{11}$ & o-methoxy aniline & \\
\hline 12 & $\mathrm{Q}_{12}$ & m-methoxy aniline & \\
\hline 13 & $\mathrm{Q}_{13}$ & p-methoxy aniline & \\
\hline 14 & $\mathrm{Q}_{14}$ & o-hydroxy aniline & \\
\hline 15 & $\mathrm{Q}_{15}$ & m-hydroxy aniline & \\
\hline 16 & $\mathrm{Q}_{16}$ & p-hydroxy aniline & \\
\hline
\end{tabular}

Table 1: List of various aromatic amines.

acetic anhydride (0.04 moles in slight excess) and refluxed on sand bath under anhydrous condition for $1 \mathrm{hr}$. Then the reaction mixture was poured into ice cold water and the crude product was filtered and dried. The dried crude product was recrystallized from ethanol. Yield: $81.34 \%$ M.P.: $188-190^{\circ} \mathrm{C}$.

Step-II: General procedure for the synthesis of 4-[6-chloro2-methyl-4-oxo quinazolin-3(4H)-yl] benzoic acid (Intermediate-II)

5-Chloro-N-acetyl anthranilic acid (0.01 moles) was added to a mixture of 4-Amino benzoic acid ( 0.02 moles), Phosphorus pentoxide ( 0.03 moles) and Glacial acetic acid $(15 \mathrm{ml})$ and the mixture was refluxed under anhydrous condition for $6 \mathrm{hrs}$. Then the reaction mixture was poured into $10 \%$ Sodium bicarbonate solution $(50 \mathrm{ml})$ and crude product was filtered and dried. The dried crude product was recrystallized from ethanol. Yield: $76.67 \%$ M.P.: 220 $-222^{\circ} \mathrm{C}$.

Step-III: General procedure for the synthesis of various derivatives of quinazolinone by mannich reaction $(Q 1$ - Q16) 4-[6-chloro-2-arylaminoethyl-4-oxoquinazolin-3(4H)-yl] benzoic acid

A mixture of 4-[6-Chloro-2-methyl-4-oxoquinazolin-3(4H)-yl] benzoic acid ( 0.01 mole), various aromatic amines ( 0.02 mole) and formaldehyde $(0.02$ mole) were taken in methanol $(80 \mathrm{ml})$ and the reaction mixture was refluxed for $4 \mathrm{hrs}$. The completion of reaction was monitored by TLC. The excess of the solvent was distilled off and the residue was recrystallized from acetone to give final product
Q1: 4-(6-chloro-4-oxo-2-(2-(phenylamino)ethyl)quinazolin$3(4 \mathrm{H})-y l)$ benzoic acid

Dark brown colored solid, Molecular formula: $\mathrm{C}_{23} \mathrm{H}_{18} \mathrm{ClN}_{3} \mathrm{O}_{3}$, Molecular weight: 419.86 , Yield: $69.22 \%$, M.P.: $176-178^{\circ} \mathrm{C}$, $\mathrm{R}_{\mathrm{f}}$ value: 0.79, FT-IR ( $\mathrm{KBr}, \mathrm{cm}^{-1}$ ): 3407.07 (N-H Str), 2905.12 (C-H Str.), 1609.91 (C=C Str.), 1711.94 (C=O Str.), 1250.04 (C-N Str.), 734.56 (Ar C-H Bend.). ${ }^{1} \mathrm{H}-\mathrm{NMR}\left(400 \mathrm{MHz}\right.$, DMSO, $\delta \mathrm{ppm}$ ): 1.55 (t, 2H, $\mathrm{CH}_{2}$ ), $3.22\left(\mathrm{q}, 2 \mathrm{H}, \mathrm{CH}_{2}\right), 4.13(\mathrm{t}, 1 \mathrm{H}, \mathrm{NH}), 6.38$ - $8.10(\mathrm{~m}, 12 \mathrm{H}, \mathrm{Ar} \mathrm{H}), 11.10$ (s, $1 \mathrm{H}, \mathrm{COOH}$ ). Mass Spectra: m/z: $421.67\left(\mathrm{M}^{+2}\right)$. Elemental Analysis, \% found (\% required): C 65.64 (65.79); H 4.28 (4.32); N 9.93 (10.01); 011.32 (11.43); Cl 8.38 (8.44).

Q2: 4-(6-chloro-2-(2-(2-nitrophenylamino)ethyl)-4-oxoquinazolin-3(4H)-yl) benzoic acid

Yellowish brown colored solid, Molecular formula: $\mathrm{C}_{23} \mathrm{H}_{17} \mathrm{ClN}_{4} \mathrm{O}_{5}$, Molecular weight: 464.86 , Yield: $68.32 \%$, M.P.: $152-154^{\circ} \mathrm{C}, \mathrm{R}_{\mathrm{f}}$ value: 0.79, FT-IR ( $\mathrm{KBr}, \mathrm{cm}^{-1}$ ): 3434.67 (N-H Str.), 2991.28 (C-H Str.), 1629.41 (C=C Str.), 1701.03 (C=0 Str.), 1254.04 (C-N Str.), 743.35 (Ar C-H Bend.), 1497.09 (Ar N=O Str.). ${ }^{1} \mathrm{H}-\mathrm{NMR}(400 \mathrm{MHz}$, DMSO, $\delta$ ppm): $1.57\left(\mathrm{t}, 2 \mathrm{H}, \mathrm{CH}_{2}\right), 3.31\left(\mathrm{q}, 2 \mathrm{H}, \mathrm{CH}_{2}\right), 4.20(\mathrm{t}, 1 \mathrm{H}, \mathrm{NH}), 6.70-$ $8.14(\mathrm{~m}, 11 \mathrm{H}, \mathrm{Ar} \mathrm{H}), 11.12$ (s, 1H, COOH). Mass Spectra: m/z: 466.43 $\left(\mathrm{M}^{+2}\right)$. Elemental Analysis, \% found (\% required): C 59.34 (59.43); H 3.65 (3.69); N 11.96 (12.05); 017.15 (17.21); Cl 7.55 (7.63).

Q3: 4-(6-chloro-2-(2-(3-nitrophenylamino)ethyl)-4-oxoquinazolin-3(4H)-yl) benzoic acid

Creamish yellow colored solid, Molecular formula: $\mathrm{C}_{23} \mathrm{H}_{17} \mathrm{ClN}_{4} \mathrm{O}_{5}$, Molecular weight: 464.86 , Yield: $67.22 \%$, M.P.: $168-170^{\circ} \mathrm{C}, \mathrm{R}_{\mathrm{f}}$ value: 0.80, FT-IR ( $\mathrm{KBr}, \mathrm{cm}^{-1}$ ): 3396.21 (N-H Str.), 2895.47 (C-H Str.), 
1599.85 (C=C Str.), 1704.19 (C=0 Str.), 1222.75 (C-N Str.), 742.59 (Ar C-H Bend.), 1452.39 (Ar N=O Str.). ${ }^{1} \mathrm{H}-\mathrm{NMR}$ (400 MHz, DMSO, $\delta$ ppm): $1.58\left(\mathrm{t}, 2 \mathrm{H}, \mathrm{CH}_{2}\right), 3.29\left(\mathrm{q}, 2 \mathrm{H}, \mathrm{CH}_{2}\right), 4.17(\mathrm{t}, 1 \mathrm{H}, \mathrm{NH}), 6.75$ - $8.12(\mathrm{~m}, 11 \mathrm{H}, \mathrm{Ar} \mathrm{H}), 11.00$ (s, 1H, COOH). Mass Spectra: m/z: $466.57\left(\mathrm{M}^{+2}\right)$. Elemental Analysis, \% found (\% required): C 59.35 (59.43); H 3.51 (3.69); N 12.08 (12.05); 017.12 (17.21); Cl 7.52 (7.63).

Q4: 4-(6-chloro-2-(2-(4-nitrophenylamino)ethyl)-4-oxoquinazolin-3(4H)-yl) benzoic acid

Pale yellow colored solid, Molecular formula: $\mathrm{C}_{23} \mathrm{H}_{17} \mathrm{ClN}_{4} \mathrm{O}_{5}$, Molecular weight: 464.86 , Yield: $67.77 \%$, M.P.: $178-180^{\circ} \mathrm{C}, \mathrm{R}_{\mathrm{f}}$ value: 0.76, FT-IR (KBr, cm${ }^{-1}$ ): 3434.65 (N-H Str.), 2917.59 (C-H Str.), 1657.18 (C=C Str.), 1754.78 (C=0 Str.), 1259.94 (C-N Str.), 796.20 (Ar C-H Bend.), 1470.73 (Ar N=O Str.). ${ }^{1} \mathrm{H}-\mathrm{NMR}(400 \mathrm{MHz}$, DMSO, $\delta$ ppm): 1.61 (t, 2H, $\left.\mathrm{CH}_{2}\right), 3.15$ (q, 2H, $\mathrm{CH}_{2}$ ), $4.11(\mathrm{t}, 1 \mathrm{H}, \mathrm{NH}), 6.65$ - $8.12(\mathrm{~m}, 11 \mathrm{H}, \mathrm{Ar} \mathrm{H}), 11.15$ (s, 1H, COOH). Elemental Analysis, \% found (\% required): C 59.37 (59.43); H 3.61 (3.69); N 11.95 (12.05); 0 17.13 (17.21); Cl 7.60 (7.63).

Q5:4-(2-(2-(2-bromophenylamino)ethyl)-6-chloro-4-oxoquinazolin-3(4H)-yl) benzoic acid

Pale red colored solid, Molecular formula: $\mathrm{C}_{23} \mathrm{H}_{17} \mathrm{BrClN}_{3} \mathrm{O}_{3}$, Molecular weight: 498.76 , Yield: $73.24 \%$, M.P.: $155-157^{\circ} \mathrm{C}, \mathrm{R}_{\mathrm{f}}$ value: 0.76, FT-IR (KBr, cm${ }^{-1}$ ): 3363.76 (N-H Str.), 2898.37 (C-H Str.), 1599.58 (C=C Str.), 1679.43 (C=0 Str.), 1258.02 (C-N Str.), 768.37 (Ar C-H Bend.), 678.46 (Ar C-Br Bend.). ${ }^{1} \mathrm{H}-\mathrm{NMR}$ (400 MHz, DMSO, $\delta$ ppm): $1.63\left(\mathrm{t}, 2 \mathrm{H}, \mathrm{CH}_{2}\right), 3.12\left(\mathrm{q}, 2 \mathrm{H}, \mathrm{CH}_{2}\right), 4.14(\mathrm{t}, 1 \mathrm{H}, \mathrm{NH}), 6.34$ - $8.11(\mathrm{~m}, 11 \mathrm{H}, \mathrm{Ar} \mathrm{H}), 11.10$ (s, 1H, COOH). Mass Spectra: m/z: $500.07\left(\mathrm{M}^{+2}\right)$. Elemental Analysis, \% found (\% required): C 55.32 (55.39); H 3.40 (3.44); N 8.35 (8.42); 09.57 (9.62); Cl 7.07 (7.11); Br 15.99 (16.02).

Q6:4-(2-(2-(3-bromophenylamino)ethyl)-6-chloro-4-oxoquinazolin-3(4H)-yl) benzoic acid

Light red colored solid, Molecular formula: $\mathrm{C}_{23} \mathrm{H}_{17} \mathrm{BrClN}_{3} \mathrm{O}_{3}$, Molecular weight: 498.76 , Yield: $72.84 \%$, M.P.: $158-160^{\circ} \mathrm{C}, \mathrm{R}_{\mathrm{f}}$ value: 0.74, FT-IR (KBr, cm ${ }^{-1}$ ): 3320.88 (N-H Str.), 2809.58 (C-H Str.), 1589.43 (C=C Str.), 1666.88 (C=0 Str.), 1258.46 (C-N Str.), 718.27 (Ar C-H Bend.), 650.43 (Ar C-Br Bend.). ${ }^{1} \mathrm{H}-\mathrm{NMR}$ (400 MHz, DMSO, $\delta$ ppm): $1.64\left(\mathrm{t}, 2 \mathrm{H}, \mathrm{CH}_{2}\right), 3.10\left(\mathrm{q}, 2 \mathrm{H}, \mathrm{CH}_{2}\right), 4.16(\mathrm{t}, 1 \mathrm{H}, \mathrm{NH}), 6.37$ - $8.13(\mathrm{~m}, 11 \mathrm{H}, \mathrm{Ar} \mathrm{H}), 11.08$ (s, $1 \mathrm{H}, \mathrm{COOH})$. Mass Spectra: m/z: $500.04\left(\mathrm{M}^{+2}\right)$. Elemental Analysis, \% found (\% required): C 55.32 (55.39); H 3.38 (3.44); N 8.36 (8.42); 09.57 (9.62); Cl 7.10 (7.11); Br 15.98 (16.02).

Q7:4-(2-(2-(4-bromophenylamino)ethyl)-6-chloro-4-oxoquinazolin-3(4H)-yl) benzoic acid

Greyish red colored solid, Molecular formula: $\mathrm{C}_{23} \mathrm{H}_{17} \mathrm{BrClN}_{3} \mathrm{O}_{3}$, Molecular weight: 498.76 , Yield: $70.24 \%$, M.P.: $160-162^{\circ} \mathrm{C}, \mathrm{R}_{\mathrm{f}}$ value: 0.70, FT-IR ( $\mathrm{KBr}, \mathrm{cm}^{-1}$ ): 3334.67 (N-H Str.), 2849.31 (C-H Str.), 1597.36 (C=C Str.), 1693.03 (C=0 Str.), 1255.20 (C-N Str.), 717.26 (Ar C-H Bend.), 637.77 (Ar C-Br Bend.). ${ }^{1} \mathrm{H}-\mathrm{NMR}(400 \mathrm{MHz}$, DMSO, $\delta$ ppm): $1.57\left(\mathrm{t}, 2 \mathrm{H}, \mathrm{CH}_{2}\right), 3.14\left(\mathrm{q}, 2 \mathrm{H}, \mathrm{CH}_{2}\right), 3.94(\mathrm{t}, 1 \mathrm{H}, \mathrm{NH}), 6.29$ - $8.10(\mathrm{~m}, 11 \mathrm{H}, \mathrm{Ar} \mathrm{H}), 11.05$ (s, 1H, COOH). Elemental Analysis, \% found (\% required): C 55.36 (55.39); H 3.41 (3.44); N 8.34 (8.42); O 9.59 (9.62); Cl 7.02 (7.11); Br 15.94 (16.02).

Q8:4-(6-chloro-2-(2-(2-chlorophenylamino)ethyl)-4-oxoquinazolin-3(4H)-yl) benzoic acid

Dark Brown colored solid, Molecular formula: $\mathrm{C}_{23} \mathrm{H}_{17} \mathrm{Cl}_{2} \mathrm{~N}_{3} \mathrm{O}_{3}$ Molecular weight: 454.31, Yield: $69.30 \%$, M.P.: $204-206^{\circ} \mathrm{C}, \mathrm{R}_{\mathrm{f}}$ value: 0.71, FT-IR (KBr, $\mathrm{cm}^{-1}$ ): 3371.64 (N-H Str.), 2863.85 (C-H Str.), 1572.74 (C=C Str.), 1711.10 (C=0 Str.), 1259.33 (C-N Str.), 713.32 (Ar C-H Bend.), 654.23 (Ar C-Cl Bend.). ${ }^{1} \mathrm{H}-\mathrm{NMR}$ (400 MHz, DMSO, $\delta$ ppm): $1.59\left(\mathrm{t}, 2 \mathrm{H}, \mathrm{CH}_{2}\right), 2.98\left(\mathrm{q}, 2 \mathrm{H}, \mathrm{CH}_{2}\right), 4.03(\mathrm{t}, 1 \mathrm{H}, \mathrm{NH}), 6.37$ $8.12(\mathrm{~m}, 11 \mathrm{H}, \mathrm{Ar} \mathrm{H}), 11.02$ (s, 1H, COOH). Mass Spectra: m/z: 456.39 $\left(\mathrm{M}^{+2}\right)$. Elemental Analysis, \% found (\% required): C 60.77 (60.81); H 3.72 (3.77); N 9.20 (9.25); 010.51 (10.57); Cl 15.58 (15.61).

Q9:4-(6-chloro-2-(2-(3-chlorophenylamino)ethyl)-4-oxoquinazolin-3(4H)-yl) benzoic acid

Pale Brown colored solid, Molecular formula: $\mathrm{C}_{23} \mathrm{H}_{17} \mathrm{Cl}_{2} \mathrm{~N}_{3} \mathrm{O}_{3}$, Molecular weight: 454.31 , Yield: $67.84 \%$, M.P.: $210-212^{\circ} \mathrm{C}, \mathrm{R}_{\mathrm{f}}$ value: 0.78, FT-IR (KBr, cm ${ }^{-1}$ ): 3394.98 (N-H Str.), 2858.37 (C-H Str.), 1504.84 (C=C Str.), 1724.98 (C=0 Str.), 1209.33 (C-N Str.), 710.11 (Ar C-H Bend.), 673.29 (Ar C-Cl Bend.). ${ }^{1} \mathrm{H}-\mathrm{NMR}$ (400 MHz, DMSO, $\delta$ ppm): $1.62\left(\mathrm{t}, 2 \mathrm{H}, \mathrm{CH}_{2}\right), 3.11\left(\mathrm{q}, 2 \mathrm{H}, \mathrm{CH}_{2}\right), 4.08(\mathrm{t}, 1 \mathrm{H}, \mathrm{NH}), 6.30$ - 8.07 (m, 11H, Ar H), 11.01 (s, 1H, COOH). Elemental Analysis, \% found (\% required): C 60.77 (60.81); H 3.71 (3.77); N 9.22 (9.25); O 10.54 (10.57); Cl 15.58 (15.61).

Q10: 4-(6-chloro-2-(2-(4-chlorophenylamino)ethyl)-4-oxoquinazolin-3(4H)-yl) benzoic acid

Creamish Brown colored solid, Molecular formula: $\mathrm{C}_{23} \mathrm{H}_{17} \mathrm{Cl}$ ${ }_{2} \mathrm{~N}_{3} \mathrm{O}_{3}$, Molecular weight: 454.31, Yield: 66.67\%, M.P.: $209-211^{\circ} \mathrm{C}$, $\mathrm{R}_{\mathrm{f}}$ value: 0.69, FT-IR (KBr, cm${ }^{-1}$ ): 3375.68 (N-H Str.), 2719.68 (C-H Str.), 1531.28 (C=C Str.), 1717.59 (C=0 Str.), 1207.04 (C-N Str.), 761.10 (Ar C-H Bend.), 640.39 (Ar C-Cl Bend.). ${ }^{1} \mathrm{H}-\mathrm{NMR}$ (400 MHz, DMSO, $\delta$ ppm): $1.60\left(\mathrm{t}, 2 \mathrm{H}, \mathrm{CH}_{2}\right), 3.17\left(\mathrm{q}, 2 \mathrm{H}, \mathrm{CH}_{2}\right), 4.10(\mathrm{t}, 1 \mathrm{H}, \mathrm{NH})$, 6.35 - $8.11(\mathrm{~m}, 11 \mathrm{H}, \mathrm{Ar} \mathrm{H}), 10.89$ (s, 1H, COOH). Mass Spectra: m/z: $456.69\left(\mathrm{M}^{+2}\right)$. Elemental Analysis, \% found (\% required): C 60.78 (60.81); H 3.74 (3.77); N 9.19 (9.25); O 10.50 (10.57); Cl 15.58 (15.61).

Q11:4-(6-chloro-2-(2-(2-methoxyphenylamino)ethyl)-4-oxoquinazolin-3(4H)-yl) benzoic acid

Yellowish White colored solid, Molecular formula: $\mathrm{C}_{24} \mathrm{H}_{20} \mathrm{ClN}_{3} \mathrm{O}_{4}$, Molecular weight: 449.89, Yield: $64.54 \%$, M.P.: $147-149^{\circ} \mathrm{C}, \mathrm{R}_{\mathrm{f}}$ value: 0.65, FT-IR (KBr, cm${ }^{-1}$ ): 3369.95 (N-H Str.), 2809.33 (C-H Str.), 1517.09 (C=C Str.), 1694.15 (C=0 Str.), 1217.93 (C-N Str.), 710.77 (Ar C-H Bend.). ${ }^{1} \mathrm{H}-\mathrm{NMR}$ (400 MHz, DMSO, $\delta \mathrm{ppm}$ ): $1.61\left(\mathrm{t}, 2 \mathrm{H}, \mathrm{CH}_{2}\right.$ ), $3.22\left(\mathrm{q}, 2 \mathrm{H}, \mathrm{CH}_{2}\right), 4.16(\mathrm{t}, 1 \mathrm{H}, \mathrm{NH}), 3.71\left(\mathrm{~s}, 3 \mathrm{H}, \mathrm{OCH}_{3}\right), 6.31-8.09(\mathrm{~m}$, 11H, Ar H), 11.04 (s, 1H, COOH). Mass Spectra: m/z: $451.27\left(\mathrm{M}^{+2}\right)$. Elemental Analysis, \% found (\% required): C 63.98 (64.07); H 4.45 (4.48); N 9.30 (9.34); 014.19 (14.23); Cl 7.84 (7.88).

Q12:4-(6-chloro-2-(2-(3-methoxyphenylamino)ethyl)-4-oxoquinazolin-3(4H)-yl) benzoic acid

Creamish White colored solid, Molecular formula: $\mathrm{C}_{24} \mathrm{H}_{20} \mathrm{ClN}_{3} \mathrm{O}_{4}$ Molecular weight: 449.89, Yield: $60.53 \%$, M.P.: $152-154^{\circ} \mathrm{C}, \mathrm{R}_{\mathrm{f}}$ val- 
ue: 0.68, FT-IR (KBr, cm ${ }^{-1}$ ): 3396.75 (N-H Str.), 2898.47 (C-H Str.), 1531.07 (C=C Str.), 1704.58 (C=0 Str.), 1239.75 (C-N Str.), 719.43 (Ar C-H Bend.). ${ }^{1} \mathrm{H}-\mathrm{NMR}$ (400 MHz, DMSO, $\delta \mathrm{ppm}$ ): $1.58(\mathrm{t}, 2 \mathrm{H}$, $\mathrm{CH}_{2}$ ), $3.11\left(\mathrm{q}, 2 \mathrm{H}, \mathrm{CH}_{2}\right), 4.13(\mathrm{t}, 1 \mathrm{H}, \mathrm{NH}), 3.75\left(\mathrm{~s}, 3 \mathrm{H}, \mathrm{OCH}_{3}\right), 5.91$ - 8.0 (m, 11H, Ar H), 11.01 (s, 1H, COOH). Elemental Analysis, \% found (\% required): C 63.99 (64.07); H 4.45 (4.48); N 9.32 (9.34); O 14.19 (14.23); Cl 7.81 (7.88).

Q13: 4-(6-chloro-2-(2-(4-methoxyphenylamino)ethyl)-4-oxoquinazolin-3(4H)-yl) benzoic acid

White Brown colored solid, Molecular formula: $\mathrm{C}_{24} \mathrm{H}_{20} \mathrm{ClN}_{3} \mathrm{O}_{4}$, Molecular weight: 449.89 , Yield: $70.79 \%$, M.P.: $138-140^{\circ} \mathrm{C}, \mathrm{R}_{\mathrm{f}}$ value: 0.66, FT-IR (KBr, cm ${ }^{-1}$ ): 3421.07 (N-H Str.), 2918.12 (C-H Str.), 1546.13 (C=C Str.), 1719.41 (C=O Str.), 1208.08 (C-N Str.), 735.04 (Ar C-H Bend.). ${ }^{1} \mathrm{H}-\mathrm{NMR}$ (400 MHz, DMSO, $\delta \mathrm{ppm}$ ): $1.64\left(\mathrm{t}, 2 \mathrm{H}, \mathrm{CH}_{2}\right.$ ), 3.14 (q, 2H, $\mathrm{CH}_{2}$ ), 4.07 (t, 1H, NH), 3.72 (s, 3H, $\mathrm{OCH}_{3}$ ), 6.30-8.03 (m, 11H, Ar H), 10.86 (s, 1H, COOH). Mass Spectra: m/z: $451.35\left(\mathrm{M}^{+2}\right)$. Elemental Analysis, \% found (\% required): C 64.01 (64.07); H 4.46 (4.48); N 9.30 (9.34); 014.18 (14.23); Cl 7.80 (7.88).

Q14: 4-(6-chloro-2-(2-(2-hydroxyphenylamino)ethyl)-4-oxoquinazolin-3(4H)-yl) benzoic acid

Greyish Black colored solid, Molecular formula: $\mathrm{C}_{23} \mathrm{H}_{18} \mathrm{ClN}_{3} \mathrm{O}_{4}$, Molecular weight: 435.86, Yield: $72.11 \%$, M.P.: $133-135^{\circ} \mathrm{C}, \mathrm{R}_{\mathrm{f}}$ value: 0.71, FT-IR (KBr, cm${ }^{-1}$ ): 3478.22 (N-H Str.), 2934.89 (C-H Str.), 1530.86 (C=C Str.), 1643.50 (C=O Str.), 1209.79 (C-N Str.), 737.35 (Ar C-H Bend.), 3446.18 (Ar C-OH Str.). ${ }^{1} \mathrm{H}-\mathrm{NMR}(400 \mathrm{MHz}$, DMSO, $\delta$ ppm): $1.69\left(\mathrm{t}, 2 \mathrm{H}, \mathrm{CH}_{2}\right), 3.25\left(\mathrm{q}, 2 \mathrm{H}, \mathrm{CH}_{2}\right), 4.01(\mathrm{t}, 1 \mathrm{H}, \mathrm{NH})$, 5.10 (s, 1H, OH), $6.24-8.12(\mathrm{~m}, 11 \mathrm{H}, \mathrm{Ar} \mathrm{H}), 11.12$ (s, 1H, COOH). Mass Spectra: m/z: $437.11\left(\mathrm{M}^{+2}\right)$. Elemental Analysis, \% found $(\%$ required): C 63.35 (63.38); H 4.10 (4.16); N 9.59 (9.64); 014.66 (14.68); Cl 8.09 (8.13).

Q15: 4-(6-chloro-2-(2-(3-hydroxyphenylamino)ethyl)-4-oxoquinazolin-3(4H)-yl) benzoic acid

Yellowish Black colored solid, Molecular formula: $\mathrm{C}_{23} \mathrm{H}_{18} \mathrm{ClN}_{3} \mathrm{O}_{4}$, Molecular weight: 435.86 , Yield: $74.38 \%$, M.P.: $138-140^{\circ} \mathrm{C}, \mathrm{R}_{\mathrm{f}}$ value: 0.74, FT-IR (KBr, cm${ }^{-1}$ ): 3477.38 (N-H Str.), 2979.13 (C-H Str.), 1531.84 (C=C Str.), 1622.98 (C=O Str.), 1207.57 (C-N Str.), 762.11 (Ar C-H Bend.), 3446.93 (Ar C-OH Str.). ${ }^{1} \mathrm{H}-\mathrm{NMR}$ (400 MHz, DMSO, $\delta$ ppm): $1.65\left(\mathrm{t}, 2 \mathrm{H}, \mathrm{CH}_{2}\right), 3.19\left(\mathrm{q}, 2 \mathrm{H}, \mathrm{CH}_{2}\right), 4.04(\mathrm{t}, 1 \mathrm{H}, \mathrm{NH}), 5.07(\mathrm{~s}$, $1 \mathrm{H}, \mathrm{OH}), 5.89-8.14(\mathrm{~m}, 11 \mathrm{H}, \mathrm{Ar} \mathrm{H}), 11.03$ (s, 1H, COOH). Elemental Analysis, \% found (\% required): C 63.33 (63.38); H 4.11 (4.16); N 9.60 (9.64); 014.63 (14.68); Cl 8.08 (8.13).

Q16: 4-(6-chloro-2-(2-(4-hydroxyphenylamino)ethyl)-4-oxoquinazolin-3(4H)-yl) benzoic acid

Black Red colored solid, Molecular formula: $\mathrm{C}_{23} \mathrm{H}_{18} \mathrm{ClN}_{3} \mathrm{O}_{4}$, Molecular weight: 435.86 , Yield: $70.25 \%$, M.P.: $144-146^{\circ} \mathrm{C}, \mathrm{R}_{\mathrm{f}}$ value: 0.69, FT-IR (KBr, cm${ }^{-1}$ ): 3377.38 (N-H Str.), 2979.49 (C-H Str.), 1572.38 (C=C Str.), 1617.87 (C=O Str.), 1249.85 (C-N Str.), 733.59 (Ar C-H Bend.), 3315.87 (Ar C-OH Str.). ${ }^{1} \mathrm{H}-\mathrm{NMR}$ (400 MHz, DMSO, $\delta$ ppm): $1.63\left(\mathrm{t}, 2 \mathrm{H}, \mathrm{CH}_{2}\right), 3.18\left(\mathrm{q}, 2 \mathrm{H}, \mathrm{CH}_{2}\right), 4.11(\mathrm{t}, 1 \mathrm{H}, \mathrm{NH}), 5.02(\mathrm{~s}$, $1 \mathrm{H}, \mathrm{OH}), 6.25-8.11(\mathrm{~m}, 11 \mathrm{H}, \mathrm{Ar} \mathrm{H}), 11.10$ (s, 1H, COOH). Elemental Analysis, \% found (\% required): C 63.31 (63.38); H 4.11 (4.16); N 9.60 (9.64); 014.66 (14.68); Cl 8.07 (8.13).
Biological study

Evaluation of analgesic activity [15-17]

Animals

Adult male albino mice (20 - 25g) were used for studying the analgesic activity. The animals (five per cage) were maintained under standard laboratory conditions (light period of $12 \mathrm{hrs} /$ day, temperature $27 \pm 2{ }^{\circ} \mathrm{C}$ with relative humidity of $45-55 \%$ ). They were fed with standard animal feed and water ad libitum. The experimental procedures were carried out in strict compliance with the Institutional Animal Ethics Committee. All experiments were performed in the morning according to the guidelines for the care of laboratory animals.

The hot-plate method: Analgesic activity of the tested compounds was determined by the hot-plate method. A total number of 90 mice were divided into 18 groups of five animals each. The first group was administered DMSO orally ( $0.2 \mathrm{ml} / \mathrm{mice})$ and kept as negative control. Ibuprofen was given as standard drug $(50 \mathrm{mg} / \mathrm{kg})$ to the second group and the tested compounds $Q_{1}$ to $Q_{16}$ dissolved in DMSO were administered at a dose of $100 \mathrm{mg} / \mathrm{kg}$ body weight to the rest of the groups. Each animal was placed individually on a hot plate and maintained at $55^{\circ} \mathrm{C}$. The time taken by the animals to lick the hind paw or jump out of the plate was taken as the reaction time, which was measured at $30 \mathrm{~min} ., 1 \mathrm{hrs}, 2 \mathrm{hrs}$ and $3 \mathrm{hrs}$. A cut off period of 30s was considered as maximal latency to avoid paw injury. The pain inhibition percentage (PIP) was calculated according to the following formula:

Pain inhibition percentage $(\mathrm{PIP})=\left(\mathrm{T}_{\mathrm{t}}-\mathrm{T}_{\mathrm{c}} / \mathrm{T}_{\mathrm{c}}\right) \times 100$

Where $T_{c}$ and $T_{t}$ are the latency for the control and drug-treated animal groups.

The acetic acid-induced writhing test: This test was conducted using the method described by Collier., et al [18]. Muscle contractions were induced in 18 groups of mice (five animals per group) by intraperitoneal injection of $0.6 \%$ solution of acetic acid (10 ml/ $\mathrm{kg}$ ). Thirty minutes before this administration, the animals in the first group were treated orally with DMSO ( $0.2 \mathrm{ml} /$ mice $)$ and they served as negative controls. Ibuprofen as the reference standard $\left(50 \mathrm{mg} / \mathrm{kg}\right.$ ) and the tested compounds $Q_{1}$ to $Q_{16}$ dissolved in DMSO were administered orally (100 mg/kg) to the animals of the rest of the groups. Immediately after administration of acetic acid the animals were placed in glass cages and the number of 'stretching' per animal was recorded during the course of the next 15 minutes. Writhing movement was accepted as contraction of the abdominal muscles accompanied by stretching of hind limbs. There was significant reduction in the number of writhes in the drug-treated animals as compared with vehicle-treated animals. This was considered a positive analgesic response and the percentage inhibition of writhing was calculated according to the following formula: \% Analgesic activity $=$ [No. of writhings for control - No. of writhings for test compounds/No. of writhings for control] $\times 100$

\section{Results and Discussion}

Chemistry: All the novel quinazolinone derivatives were synthesized, purified and separated by using column chromatography or 
recrystallization method. Synthesized compounds were characterized by using Elemental analysis, FT-IR, ${ }^{1}$ HNMR and Mass Spectrometric studies. The integration curves fully support the orientation of protons in the analyzed compounds. Furthermore, all the compounds demonstrated the characteristic chemical shifts for the quinazolinone nucleus. Additionally, synthesized compounds were analyzed by mass spectra and indicated no difference in the fragmentation pattern among the set of synthesized series.

Analgesic activity: The analgesic activity was assessed by using hot plate and acetic acid induced writhing methods using Ibuprofen as the standard drug. The analgesic activity data by hot plate method was obtained as mean latency time at $30 \mathrm{~min}$., $1 \mathrm{hrs}, 2 \mathrm{hrs}$ and $3 \mathrm{hrs}$ intervals and expressed in \% inhibition as shown in table 2 and 3. Compounds $Q_{5}, Q_{8}$ and $Q_{9}$ showed excellent analgesic activity as $89.96 \%, 88.40 \%$ and $74.92 \%$ inhibition respectively at $3^{\text {rd }}$ hrs, which were nearby $100 \%$ inhibition of the standard Ibuprofen drug used and also greater than the other quinazolinone derivatives. Compounds $Q_{5}, Q_{8}$ and $Q_{9}$ showed significant $\%$ of analgesic activity as $90.92 \%, 88.44 \%$ and $87.97 \%$ in acetic acid induced writhing method as shown in table 4.

\begin{tabular}{|l|c|c|c|c|}
\hline \multirow{2}{*}{$\begin{array}{c}\text { Com- } \\
\text { pounds }\end{array}$} & \multicolumn{4}{|c|}{ Mean Latency Time (s) \pm SEM } \\
\cline { 2 - 6 } & $\mathbf{0 . 5} \mathbf{~ h r}$ & $\mathbf{1 ~ h r}$ & $\mathbf{2} \mathbf{~ h r}$ & $\mathbf{3 ~ h r}$ \\
\hline Control & $2.49 \pm 0.010$ & $2.57 \pm 0.012$ & $3.08 \pm 0.008$ & $3.19 \pm 0.013$ \\
\hline Ibuprofen & $3.49 \pm 0.015$ & $4.02 \pm 0.017$ & $5.43 \pm 0.017$ & $6.38 \pm 0.016^{* * *}$ \\
\hline$Q_{1}$ & $3.01 \pm 0.011$ & $3.42 \pm 0.012$ & $4.59 \pm 0.009$ & $5.40 \pm 0.010^{* *}$ \\
\hline$Q_{2}$ & $3.08 \pm 0.015$ & $3.39 \pm 0.014$ & $4.50 \pm 0.013$ & $5.22 \pm 0.013^{* *}$ \\
\hline$Q_{3}$ & $3.10 \pm 0.018$ & $3.47 \pm 0.019$ & $4.56 \pm 0.019$ & $5.29 \pm 0.014^{* *}$ \\
\hline$Q_{4}$ & $3.04 \pm 0.013$ & $3.36 \pm 0.012$ & $4.49 \pm 0.011$ & $5.16 \pm 0.012^{* *}$ \\
\hline$Q_{5}$ & $3.43 \pm 0.005$ & $4.05 \pm 0.006$ & $5.18 \pm 0.004$ & $6.06 \pm 0.007^{* * *}$ \\
\hline$Q_{6}$ & $3.28 \pm 0.018$ & $4.00 \pm 0.022$ & $5.05 \pm 0.017$ & $5.45 \pm 0.019^{* *}$ \\
\hline$Q_{7}$ & $3.06 \pm 0.014$ & $3.44 \pm 0.017$ & $4.46 \pm 0.011$ & $5.35 \pm 0.014^{* *}$ \\
\hline$Q_{8}$ & $3.47 \pm 0.007$ & $4.01 \pm 0.009$ & $5.07 \pm 0.010$ & $6.01 \pm 0.008^{* * *}$ \\
\hline$Q_{9}$ & $3.21 \pm 0.011$ & $3.59 \pm 0.008$ & $5.01 \pm 0.010$ & $5.58 \pm 0.012^{* * *}$ \\
\hline$Q_{10}$ & $3.13 \pm 0.008$ & $3.58 \pm 0.005$ & $5.03 \pm 0.006$ & $5.50 \pm 0.004^{* *}$ \\
\hline$Q_{11}$ & $3.02 \pm 0.011$ & $3.40 \pm 0.018$ & $4.40 \pm 0.009$ & $5.01 \pm 0.012^{* *}$ \\
\hline$Q_{12}$ & $3.10 \pm 0.017$ & $3.33 \pm 0.023$ & $4.35 \pm 0.019$ & $5.05 \pm 0.010^{* *}$ \\
\hline$Q_{13}$ & $3.14 \pm 0.012$ & $3.38 \pm 0.012$ & $4.52 \pm 0.017$ & $5.25 \pm 0.016^{* *}$ \\
\hline$Q_{14}$ & $3.00 \pm 0.008$ & $3.25 \pm 0.006$ & $4.31 \pm 0.012$ & $4.58 \pm 0.014^{* *}$ \\
\hline$Q_{15}$ & $3.16 \pm 0.024$ & $3.56 \pm 0.021$ & $4.58 \pm 0.020$ & $5.19 \pm 0.021^{* *}$ \\
\hline$Q_{16}$ & $3.09 \pm 0.009$ & $3.30 \pm 0.016$ & $4.30 \pm 0.013$ & $4.55 \pm 0.006^{* *}$ \\
\hline & & & & \\
\hline
\end{tabular}

Table 2: Analgesic activity of the tested compounds in mice using hot-plate method.

Values are expressed as mean \pm SEM of five animals in each group. **Statistically significant $(\mathrm{P}<0.05)$.

${ }^{* * *}$ Statistically significant $(\mathrm{P}<0.01)$.

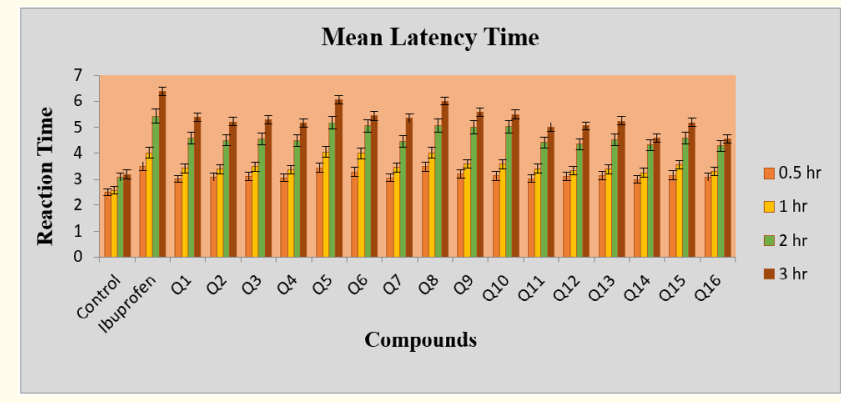

Figure 1: Effect of various treatments on mean latency time by using hot plate method.

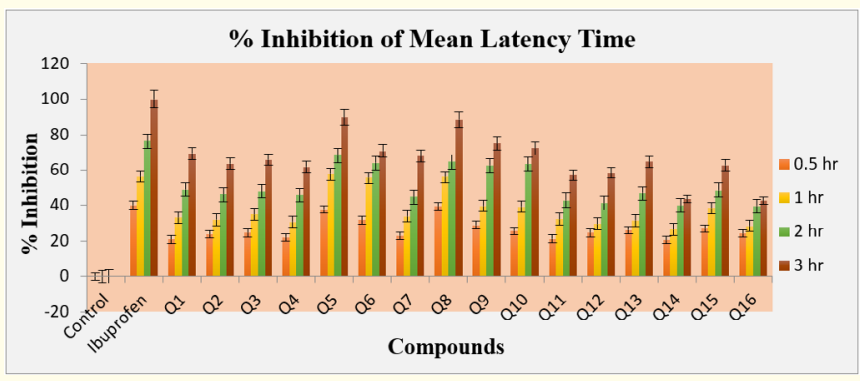

Figure 2: \% inhibition of mean latency time by using hot plate method.

\begin{tabular}{|l|c|c|c|c|}
\hline \multirow{2}{*}{ Compounds } & \multicolumn{4}{|c|}{ Pain inhibition (\%) } \\
\cline { 2 - 5 } & $\mathbf{0 . 5} \mathbf{~ h r}$ & $\mathbf{1 ~ h r}$ & $\mathbf{2} \mathbf{~ h r}$ & $\mathbf{3 ~ h r}$ \\
\hline Control & - & - & - & - \\
\hline Ibuprofen & 40.16 & 56.42 & 76.29 & $100^{* * *}$ \\
\hline $\mathrm{Q}_{1}$ & 20.88 & 33.07 & 49.02 & $69.27^{* *}$ \\
\hline $\mathrm{Q}_{2}$ & 23.69 & 31.90 & 46.10 & $63.63^{* *}$ \\
\hline $\mathrm{Q}_{3}$ & 24.49 & 35.01 & 48.05 & $65.83^{* *}$ \\
\hline $\mathrm{Q}_{4}$ & 22.08 & 30.73 & 45.77 & $61.75^{* *}$ \\
\hline $\mathrm{Q}_{5}$ & 37.75 & 57.58 & 68.18 & $89.96^{* * *}$ \\
\hline $\mathrm{Q}_{6}$ & 31.72 & 55.64 & 63.96 & $70.84^{* *}$ \\
\hline $\mathrm{Q}_{7}$ & 22.89 & 33.85 & 44.80 & $67.71^{* *}$ \\
\hline $\mathrm{Q}_{8}$ & 39.35 & 56.03 & 64.61 & $88.40^{* * *}$ \\
\hline $\mathrm{Q}_{9}$ & 28.91 & 39.68 & 62.66 & $74.92^{* * *}$ \\
\hline $\mathrm{Q}_{10}$ & 25.70 & 39.29 & 63.31 & $72.41^{* *}$ \\
\hline $\mathrm{Q}_{11}$ & 21.28 & 32.29 & 42.85 & $57.05^{* *}$ \\
\hline $\mathrm{Q}_{12}$ & 24.49 & 29.57 & 41.23 & $58.30^{* *}$ \\
\hline $\mathrm{Q}_{13}$ & 26.10 & 31.51 & 46.75 & $64.57^{* *}$ \\
\hline $\mathrm{Q}_{14}$ & 20.48 & 26.45 & 39.93 & $43.57^{* *}$ \\
\hline $\mathrm{Q}_{15}$ & 26.90 & 38.52 & 48.70 & $62.69^{* *}$ \\
\hline $\mathrm{Q}_{16}$ & 24.09 & 28.40 & 39.61 & $42.63^{* *}$ \\
\hline
\end{tabular}

Table 3: \% inhibition of analgesic activity of the tested compounds in mice using hot-plate method. 


\begin{tabular}{|l|c|c|}
\hline Compounds & $\begin{array}{c}\text { No. of writhings in 15 } \\
\text { minutes } \pm \text { SEM }\end{array}$ & $\begin{array}{c}\text { \% Analgesic } \\
\text { activity }\end{array}$ \\
\hline Control & $61.37 \pm 3.41$ & 00 \\
\hline Ibuprofen & $4.31 \pm 0.52$ & $92.97^{* * *}$ \\
\hline$Q_{1}$ & $13.31 \pm 1.18$ & $78.31^{* * *}$ \\
\hline$Q_{2}$ & $14.07 \pm 1.29$ & $77.07^{* * *}$ \\
\hline$Q_{3}$ & $11.19 \pm 1.05$ & $81.76^{* * *}$ \\
\hline$Q_{4}$ & $16.57 \pm 2.24$ & $72.99^{* *}$ \\
\hline$Q_{5}$ & $5.57 \pm 0.59$ & $90.92^{* * *}$ \\
\hline$Q_{6}$ & $12.46 \pm 1.47$ & $79.69^{* * *}$ \\
\hline$Q_{7}$ & $13.14 \pm 1.28$ & $78.58^{* *}$ \\
\hline$Q_{8}$ & $7.09 \pm 2.16$ & $88.44^{* * *}$ \\
\hline$Q_{9}$ & $7.38 \pm 2.44$ & $87.97^{* * *}$ \\
\hline$Q_{10}$ & $11.41 \pm 1.86$ & $81.40^{* * *}$ \\
\hline$Q_{11}$ & $21.46 \pm 2.11$ & $65.09^{* *}$ \\
\hline$Q_{12}$ & $18.33 \pm 1.37$ & $70.13^{* *}$ \\
\hline$Q_{13}$ & $14.45 \pm 1.26$ & $76.45^{* *}$ \\
\hline$Q_{14}$ & $20.03 \pm 2.02$ & $67.36^{* *}$ \\
\hline$Q_{15}$ & $17.41 \pm 2.47$ & $71.63^{* *}$ \\
\hline$Q_{16}$ & $23.20 \pm 2.58$ & $62.19^{* *}$ \\
\hline
\end{tabular}

Table 4: Acetic acid induced writhing response of the tested compounds and \% analgesic activity.

Values are expressed as mean \pm SEM of five animals in each group.

**Statistically significant $(\mathrm{P}<0.05)$.

${ }^{* * *}$ Statistically significant $(\mathrm{P}<0.01)$.

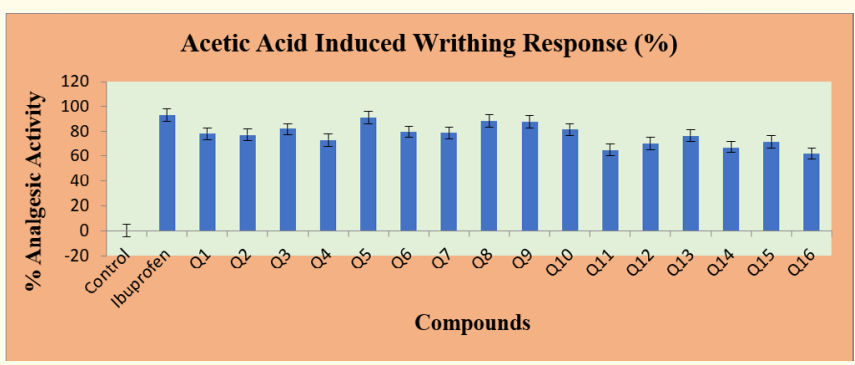

Figure 3: Acetic acid induced writhing test of compounds.

\section{Conclusion}

The main focus of this research work was to synthesize novel series of quinazolinone derivatives, purify, characterize and evaluate their analgesic activity. From the results, it can be concluded that the modified quinazolinone show significant biological evaluation as analgesic agents. However, further evaluation of quinazolinone will be undertaken, concerning the structural arrangements in ring for analgesic activity.

\section{Acknowledgement}

The authors are thankful to Indore Institute of Pharmacy, Indore for providing chemicals and facilities to conduct research work.

\section{Conflict of Interest}

The authors declared no conflict of interest.
Bibliography

1. El-Azab Adel S., et al. "Design, synthesis and biological evaluation of novel quinazoline derivatives as potential antitumor agents". European Journal of Medicinal Chemistry 45 (2010): 4188-4198.

2. Birhan Yihenew Simegniew., et al. "In vivo antimalarial evaluation of some 2,3-disubstituted-4(3H)-quinazolinone derivatives". BMC Research Notes 8 (2015): 01-06.

3. Panda Sunakar and Tripathy Upendra Prasad. “Quinazoline: A molecule of significant pharmacological and biological activity". Research Journal of Pharmacy and Technology 6.8 (2013): 849-855.

4. Kumar Ashok., et al. "Exploration of antimicrobial and antioxidant potential of newly synthesized 2,3-disubstituted quinazoline-4(3H)-ones". Bioorganic and Medicinal Chemistry Letters 21 (2011): 4353-4357.

5. Rhee Hee-Kyung., et al. "Synthesis and cytotoxicity of 2-phenylquinazolin-4(3H)-one derivatives". European Journal of Medicinal Chemistry 46 (2011): 3900-3908.

6. Krishnan Suresh Kumar., et al. "Synthesis, antiviral activity and cytotoxicity evaluation of Schiff bases of some 2-phenyl quinazoline-4(3H)-ones". European Journal of Medicinal Chemistry 45 (2010): 5474-5479.

7. Reddy PSN., et al. "Antibacterial, antifungal and antifeedant activity of quinazolinonyl- $\beta$-lactams/quinazolinones and bis (quinazolinonyl- $\beta$-lactams)". Rasayan Journal of Chemistry 03.04 (2010): 635-640.

8. Jatav Varsha., et al. "CNS depressant and anticonvulsant activities of some novel 3-[5-substituted 1,3,4-thiadiazole-2-yl]2-styryl quinazoline-4(3H)-ones". European Journal of Medicinal Chemistry 43 (2007): 1945-1954.

9. Abuelizz Hatem A., et al. "Molecular docking and anticonvulsant activity of newly synthesized quinazoline derivatives". Molecules 22 (2017): 01-13.

10. Alanazi Amer M., et al. "Synthesis, antitumor and antimicrobial activity of some new 6-methyl-3-phenyl-4(3H)-quinazolinone analogues". Journal of Enzyme Inhibition and Medicinal Chemistry 31 (2015): 721-735.

11. Kubicova Lenka., et al. "Synthesis and biological evaluation of quinazoline-4-thiones”. Molecules 8 (2003): 756-769.

12. Tyagi Mirdula., et al. "Synthesis and blood lowering activity of some newer quinazolinones". Indian Journal of Chemistry 41B (2002): 2367-2370.

13. Tiwary Bipransh Kumar., et al. "Implication of quinazoline$4(3 \mathrm{H})$-ones in medicinal chemistry". Journal of Chemical Biology and Therapeutics 1.1 (2015): 01-07. 
14. Wasfy AAF., et al. "Synthesis and anticancer properties of novel quinazoline derivatives". International Journal of Research in Pharmacy and Chemistry 5.1 (2015): 34-40.

15. Nehal A Hamdy., et al. "Potent anti-inflammatory and analgesic activities of new derivatives of chalcone, pyridine, pyrazole and isoxazole incorporated into 5,6,7,8- tetrahydronaphthalene". Egyptian Pharmaceutial Journal 11.1 (2012): 22-30.

16. B Ravi Kiran., et al. "Synthesis, evaluation of analgesic and anti-inflammatory activities of substituted 1,2-Benzoxazolone and 3-Chloro-1,2-Benzoxazole derivatives". International Journal of Pharmaceutical Science and Research 6.7 (2015): 2918-2925.

17. Kasabe Amit J., et al. "Synthesis, anti-tubercular and analgesic activity evaluation of new 3-pyrazoline derivatives". International Journal of Pharmacy and Pharmaceutical Sciences 2.2 (2010): 132-135.

18. Collier HO., et al. "The abdominal constriction response and its suppression by analgesic drug in the mouse". British Journal of Pharmacology and Chemotherapy 32 (1968): 295-310.

\section{Assets from publication with us}

- Prompt Acknowledgement after receiving the article

- Thorough Double blinded peer review

- Rapid Publication

- Issue of Publication Certificate

- High visibility of your Published work

Website: https://www.actascientific.com/

Submit Article: https://www.actascientific.com/submission.php Email us: editor@actascientific.com

Contact us: +91 9182824667 